Брагов А.М., Жегалов Д.В., Константинов А.Ю., Кочетков А.В., Модин И.А., Савихин А.О. Экспериментальное исследование деформационных свойств пакетов плетеных металлических сеток при динамическом и квазистатическом нагружении // Вестник Пермского национального исследовательского политехнического университета. Механика. 2016. - № 3. - C. 252-262. DOI: 10.15593/perm.mech/2016.3.17

Bragov A.M., Zhegalov D.V., Konstantinov A.Yu., Kochetkov A.V., Modin I.A., Savikhin A.O. Experimental study of deformation properties of a package of woven metal mesh under dynamic and quasi-static stressing. PNRPU Mechanics Bulletin. 2016. No. 3. Pp. 252-262. DOI: 10.15593/perm.mech/2016.3.17

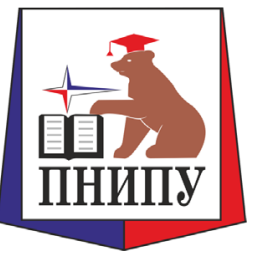

ВЕСТНИК ПНИПУ. МЕХАНИКА

№ 3, 2016

PNRPU MECHANICS BULLETIN

http://vestnik.pstu.ru/mechanics/about/inf/

DOI: $10.15593 /$ perm.mech/2016.3.17

УДК 539.3

\title{
ЭКСПЕРИМЕНТАЛЬНОЕ ИССЛЕДОВАНИЕ ДЕФОРМАЦИОННЫХ ХАРАКТЕРИСТИК ПАКЕТОВ ПЛЕТЕНЫХ МЕТАЛЛИЧЕСКИХ СЕТОК ПРИ ДИНАМИЧЕСКОМ И КВАЗИСТАТИЧЕСКОМ НАГРУЖЕНИИ
}

\author{
А.М. Брагов, Д.В. Жегалов, А.Ю. Константинов, \\ А.В. Кочетков, И.А. Модин, А.О. Савихин
}

Научно-исследовательский институт механики Национального исследовательского Нижегородского государственного университета им. Н.И. Лобачевского, Нижний Новгород, Россия

\section{O CTATbE}

Получена: 03 июня 2016 г Принята: 26 сентября 2016 г. Опубликована: 30 сентября 2016 г.

Ключевые слова:

плетеная металлическая сетка, эксперимент, разрезной стержень Гопкинсона, динамическая деформация, статическое растяжение, нелинейность, необратимость

\begin{abstract}
АННОТАЦИЯ
Многослойные газопроницаемые пакеты из металлических плетеных сеток являются перспективным демпфирующим элементом, защищающим конструкции от ударных и взрывных воздействий. За счет развитой межфазной поверхности пакеты могут отбирать значительную долю энергии горячих продуктов взрыва и снижать интенсивность проходящих ударных волн. Пакеты сеток конструктивно формируются путем свободного наложения слоев друг на друга с сохранением направлений проволок, поэтому пакеты можно считать высокопористой деформируемой средой, обладающей ортотропными свойствами. Проведены экспериментальные исследования деформационных и прочностных свойств конструктивно ортотропных пакетов плетеных металлических сеток при статическом и динамическом нагружении. Пакет сетки сопротивляется сжатию по нормали к слоям сетки: первая ось - ортотропии и растяжению вдоль направлений проволок, другие оси - ортотропии. Для ударного растяжения в плоскости слоев использовался аналог схемы Николаса, представляющей собой модификацию метода Кольского. В разрезных стержнях Гопкинсона сделаны продольные пазы, в которых размещается и закрепляется испытываемый образец. Ударное растяжение проводится в волне растяжения, формирующейся в стержнях в результате отражения от свободного торца первичной волны сжатия, которая про-
\end{abstract}

( Брагов Анатолий Михайлович - доктор технических наук, профеeccop, e-mail: bragov@mech.unn.ru Жегалов Дмитрий Владимирович - кандидат технических наук, старший научный сотрудник, e-mail: zhegalov@mech.unn.ru Константинов Александр Юрьевич - кандидат технических наук, старший научный сотрудник, e-mail: constantinov.al@yandex.ru Кочетков Анатолий Васильевич - доктор физико-математических наук, профеccop, e-mail: kochetkov@mech.unn.ru Модин Иван Александрович - аспирант, e-mail: mianet@mail.ru

Савихин Андрей Олегович - младший научный сотрудник, e-mail: andrey-savikhin@yandex.ru

Anatoliy M. Bragov - Doctor of Technical Sciences, Professor, e-mail: bragov@mech.unn.ru

Dmitriy V. Zhegalov - PhD in Technical Sciences, Senior Researcher, e-mail: zhegalov@mech.unn.ru

Aleksandr Yu. Konstantinov - PhD in Technical Sciences, Senior Researcher, e-mail: constantinov.al@yandex.ru

Anatoliy V. Kochetkov - Doctor of Physical and Mathematical Sciences, Professor, e-mail: kochetkov@mech.unn.ru

Ivan A. Modin - PhD student, e-mail: mianet@mail.ru

Andrey O. Savikhin - Junior Researcher, e-mail: andrey-savikhin@yandex.ru 
ходит через стык стержней, не деформируя образец. Статическое растяжение образцов сеток производится на испытательных машинах Zwick. Испытания проведены для образцов с различным шагом плетения сеток и различным количеством слоев. Опытами определены необходимые размеры рабочей части образца. Показано, что диаграммы деформирования при растяжении вдоль проволок в плоскости слоев и при сжатии по нормали к слоям сетки при всех режимах нагружения носят нелинейный и необратимый характер, а также проявляют существенную зависимость от скорости десормации. При квазистатическом растяжении пакетов сеток в направлении проволок существенное влияние оказывает их предварительное обжатие по нормали к слоям сеток. При динамическом растяжении этот эффект выражен значительно слабее.

\title{
EXPERIMENTAL STUDY OF DEFORMATION PROPERTIES OF A PACKAGE OF WOVEN METAL MESH UNDER DYNAMIC AND QUASI-STATIC STRESSING
}

\author{
A.M. Bragov, D.V. Zhegalov, A.Yu. Konstantinov, \\ A.V. Kochetkov, I.A. Modin, A.O. Savikhin \\ Research Institute of Mechanics, National Research Lobachevsky State University of Nizhny Novgorod, \\ Nizhny Novgorod, Russian Federation
}

\begin{tabular}{l} 
ARTICLE INFO \\
\hline Received: 03 June 2016 \\
Accepted: 26 September 2016 \\
Published: 30 September 2016 \\
\hline Keywords: \\
woven wire mesh, experiment, \\
split Hopkinson rod, dynamic \\
deformation, static stretching, \\
non-linearity, irreversibility
\end{tabular}

\begin{abstract}
The multilayer gas-permeable bags of woven metal meshes are a promising damping element that protects the structure from shock and explosive effects. Due to the developed interface, the packages may select a significant proportion of the explosion energy of hot products and reduce the intensity of shock waves. Mesh bags are constructively formed by a free deposition of layers on each other while maintaining the directions of threads, so the packages can be considered as a highly porous deformable medium having orthotropic properties. Experimental studies have been made on deformation and strength properties of structurally orthotropic packages of woven metal grids under static and dynamic stressing. Mesh Package resists compression normal to the layers of the grid, it is the first orthotropy axis, and resists the stretching along the directions of threads, it is the other orthotropy axis. For the impact stretching in the plane of the layers we used an analogue of Nicholas circuit which is a modification of the Kolskiy method. In the split Hopkinson bars we made longitudinal grooves which house and fix the test specimen. Shock stretching is carried out in the wave of stretching which emerges in the bars as a result of reflection from the free end of the primary compression wave that passes the junction of rods without deforming the sample. Static stretching of grid samples is performed using Zwick testing machines. Tests were carried out for samples with different pitch and weave meshes and a different number of layers. Experiments have determined the necessary dimensions of the working part of the sample. It is shown that the stress-strain diagram in tension along the threads in the plane of the layers and the compressive normal to the layers of the grid for all loading conditions are non-linear and irreversible, but also show a significant dependence on the strain rate. In the quasi-static tensile packages of grids in the direction of the filaments is significantly affected by their prior compression normal to the layers of mesh. Under dynamic stretching this effect is much weaker.
\end{abstract}

\section{Введение}

Разработка и анализ поведения различного вида защитных конструкций, работающих в условиях ударного и взрывного нагружения, являются важной прикладной задачей [1-8]. Многослойные газопроницаемые пакеты из металлических плетеных сеток являются перспективным демпфирующим элементом, защищающим конструкции от ударных и взрывных воздействий [9-12]. Такой пакет сетки за счет сильно развитой межфазной поверхности может отбирать значительную долю энергии горячих продуктов взрыва и снижать интенсивность проходящих ударных волн. Это приводит к уменьшению пиковых значений давления 
во взрывных камерах [9], а также к уменьшению и остаточного давления. В ряде работ [1113] даются оценки эффективности подобных многослойных экранов с точки зрения снижения газодинамических параметров проходящих ударных волн. Так как пакет сеток конструктивно формируется путем наложения слоев друг на друга, пакет можно считать высокопористой деформируемой средой, обладающей ортотропными свойствами. При использовании таких сеток, как показано в $[13,14]$, пакет сопротивляется сжатию по нормали к слоям сеток и растяжению вдоль проволок в плоскости сеток. Для рационального проектирования подобных демпфирующих элементов необходимо знать их деформационные и прочностные свойства. В [14] приведены некоторые результаты экспериментальных исследований деформационных характеристик пакетов сеток на растяжение и сжатие в направлении осей ортотропии в квазистатическом режиме нагружения. Ниже приводятся результаты новых экспериментальных исследований деформирования пакетов плетеных металлических сеток при квазистатическом и динамическом нагружении.

\section{1. Методика динамических испытаний}

Экспериментальные исследования динамических свойств пакетов плетеных металлических сеток проводились на экспериментальной установке с разрезным стержнем Гопкинсона [15-17]. Образцы изготавливались из двух типов сеток: НУ ГОСТ 3826-82 $2 \times 2 \times 0,5$ и $3,2 \times 3,2 \times 0,8$. Здесь первые две цифры - шаг плетения, последняя цифра - диаметр проволоки в миллиметрах. Оба типа сеток подобраны исходя из одинаковых значений объемного содержания металла - 0,14 и коэффициента проницаемости одного слоя 0,64. При сжатии по нормали к слоям сетки испытания проведены для двух видов образцов: кругового сечения диаметром 20 мм и квадратного сечения со стороной 50 мм. Испытывались образцы, состоящие из 10 и 20 слоев сетки, наложенных друг на друга. Направления проволок были одинаковы для всех слоев. Схемы экспериментов для различного вида образцов показаны на рис. 1 и 2. В экспериментах использовалась установка, состоящая из высокопрочных стальных стержней диаметром 20 мм.

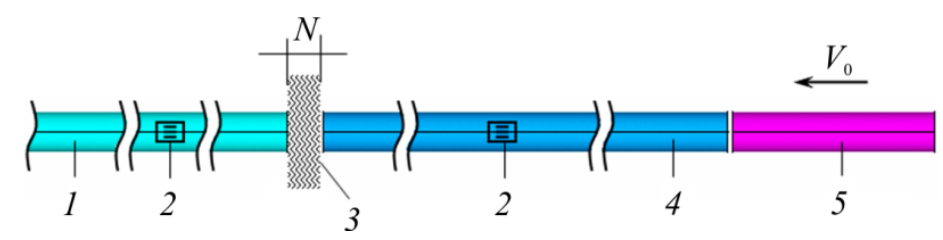

Рис. 1. Схема эксперимента с образцом квадратного сечения

Fig. 1. Experimental set up with a square section sample

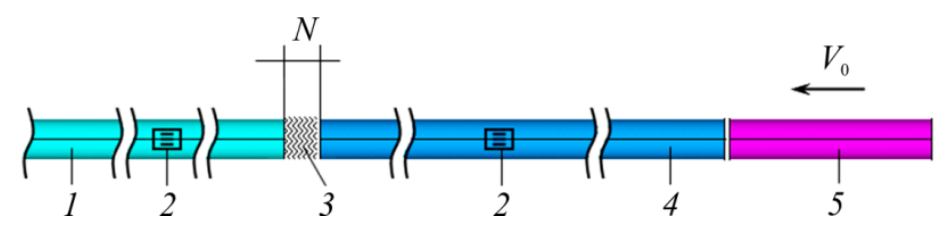

Рис. 2. Схема эксперимента с образцом круглого сечения

Fig. 2. Experimental set up with a circular section sample

Испытания проводились следующим образом. Образец 3 , состоящий из $N$ слоев металлической сетки, помещался между двумя мерными стержнями 1 (опорный) и 4 (нагружающий). Один из стержней нагружался ударом короткого стержня-ударника 5 , дви- 
жущегося со скоростью $V_{0}$. При этом в стержне 4 формировался прямоугольный импульс сжатия, который нагружал образец. В процессе эксперимента с помощью тензодатчиков 2 регистрировались деформации в сечениях нагружающего и опорного мерного стержней. Данная информация позволяет с помощью формул Кольского [15] рассчитать временную историю изменения длины образца, а также действующую на него при этом силу.

Из одномерной теории распространения упругих волн в полубесконечных стержнях известно, что деформация в волне связаны с массовой скоростью $d U / d t$ соотношениями [1]

$$
\varepsilon(t)=\frac{1}{C} \cdot \frac{d U}{d t},
$$

откуда смещение $U(t)$ частиц в волне [2]

$$
U(t)=C \int_{0}^{t} \varepsilon(t) \cdot d t
$$

Здесь $C$ - скороть звука в стержне.

На основании последней формулы можно записать перемещения торцов стержней 4 и 1 , примыкающих к образцу. Перемещение правого торца $U_{1}(t)$ складывается из перемещения $U_{1}^{I}(t)$, вызванного распространением падающего импульса $\varepsilon^{\mathrm{I}}(t)$, и перемещения $U_{1}^{R}(t)$, вызванного распространением отраженного импульса $\varepsilon^{R}(t)[3]$.

$$
U_{1}(t)=C \int_{0}^{t} \varepsilon^{I}(t) \cdot d t+(-C) \int_{0}^{t} \varepsilon^{R}(t) \cdot d t=C \int_{0}^{t}\left(\varepsilon^{I}(t)-\varepsilon^{R}(t)\right) d t
$$

Перемещение левого торца $U_{2}(t)$ вызвано распространением прошедшего импульса $\varepsilon^{T}(t)[4]:$

$$
U_{2}(t)=C \int_{0}^{t} \varepsilon^{T}(t) \cdot d t
$$

Изменение длины образца $d L$ в процессе нагружения можно расчитать следующим образом [5]:

$$
d L(t)=U_{2}(t)-U_{1}(t) .
$$

Средняя относительная деформация образца длиной $L_{0}$ рассчитывается так [6]:

$$
\varepsilon_{S}(t)=\frac{U_{2}(t)-U_{1}(t)}{L_{0}} .
$$

Если выразить ее через импульсы в стержнях [7]

$$
\varepsilon_{S}(t)=\frac{C}{L_{0}} \int_{0}^{t}\left[\varepsilon^{I}(t)-\varepsilon^{R}(t)-\varepsilon^{T}(t)\right] \cdot d t,
$$

то скорость деформации образца [8]

$$
\dot{\varepsilon}_{S}(t)=\frac{C}{L_{0}}\left(\varepsilon^{I}(t)-\varepsilon^{R}(t)-\varepsilon^{T}(t)\right) .
$$


Для нахождения напряжения в образце следует рассмотреть усилия на торцах образца. Усилие на правом торце $P_{1}(t)$ складывается из сжимающего усилия $P_{1}^{I}(t)$, вызванного импульсом $\varepsilon^{I}(t)$, а усилие на левом торце вызвано импульсом $\varepsilon^{T}(t)$. Тогда с учетом закона Гука (стержни имеют высокий предел упругости и деформируются упруго) [9]

$$
P_{1}(t)=E A\left[\varepsilon^{I}(t)+\varepsilon^{R}(t)\right] ; \quad P_{2}(t)=E A \varepsilon^{T}(t),
$$

где $E$ и $A$ - соответственно модуль Юнга и площадь поперечного сечения стержней. Средняя сила $P=\frac{P_{1}(t)+P_{2}(t)}{2}$, отсюда среднее значение напряжения в образце [10]

$$
\sigma_{s}(t)=\frac{P}{A}=\frac{E A}{2 A}\left[\varepsilon^{I}(t)-\varepsilon^{R}(t)-\varepsilon^{T}(t)\right]=\frac{E}{2}\left[\varepsilon^{I}(t)+\varepsilon^{R}(t)+\varepsilon^{T}(t)\right] .
$$

На рис. 3 показаны характерные осцилограммы деформаций. Цифрами 1-12, отмечены содержательные участки импульсов, по которым строились диаграммы напряжениядеформации. Видно, что деформирование образца проходило в несколько циклов, связанных с отражением волн от торцов стержней. В процессе эксперимента с помощью тензодатчиков регистрировались деформации в сечениях нагружающего (сверху) и опорного (снизу) мерного стержня. По этой информации рассчитывалась временная история изменения длины образца, а также действующая на него при этом сила.

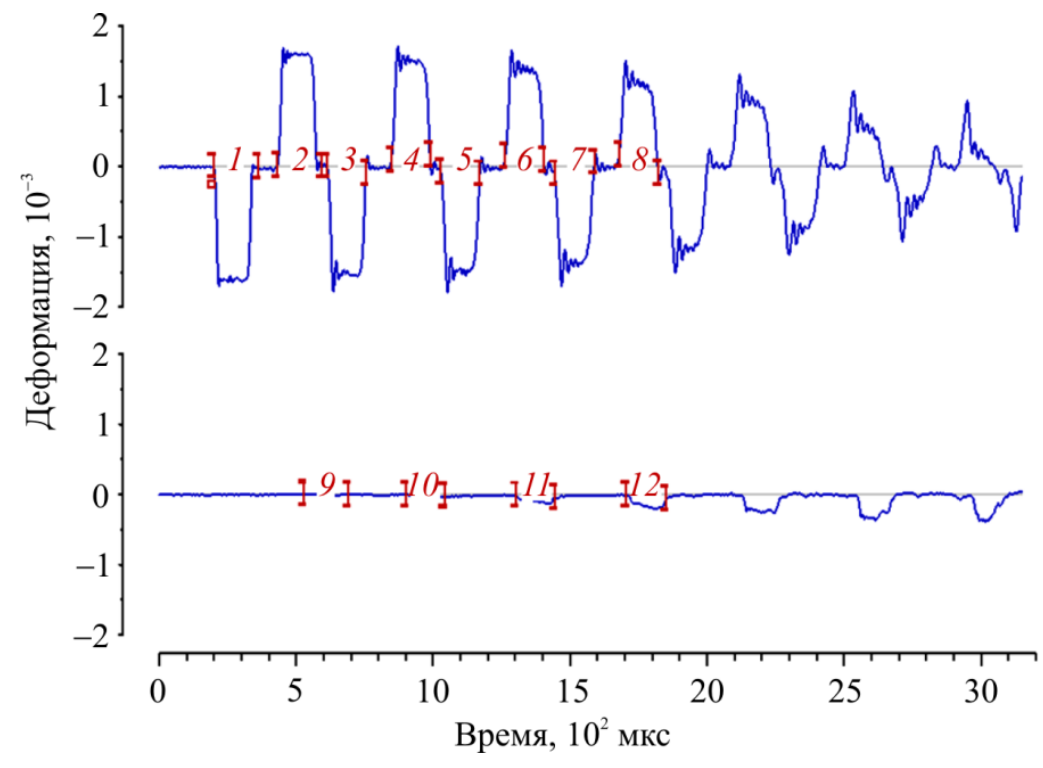

Рис. 3. Деформации в сечениях мерных стержней

Fig. 3. Deformation in sections of measuring rods

Для испытания пакета сеток в условиях ударного растяжения в плоскости слоев использовался аналог схемы Николаса [18], представляющей себой модификацию метода Кольского. Схема эксперимента показана на рис. 4. Плоский образец, предварительно обжатый по нормали к слоям сетки, закрепляется в вилках мерных стержней с помощью болтовой стяжки. Торцы стержней при этом стыкуются. Система нагружается ударником, разогнанным в пневматической пушке. Сформировавшийся при этом сжимающий импульс проходит через стык, не деформируя образец. Отражаясь от свободного торца стержня 1, этот импульс меняет знак и превращается в импульс растяжения, который 


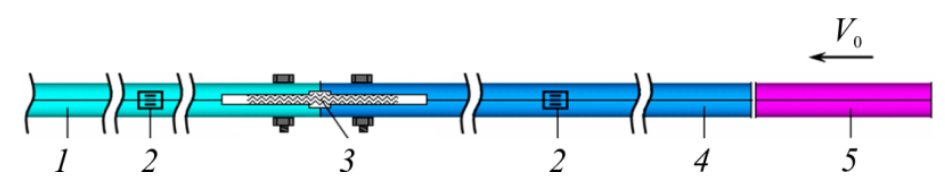

Рис. 4. Схема эксперимента на динамическое растяжение

Fig. 4. Experimental set up related to dynamic stressing

движется в сторону образца и нагружает его. Регистрация деформации в тензодатчиках 2 позволяет рассчитать деформацию образца и силу, которая действовала на образец в процессе нагружения. Использованная в экспериментах установка состояла из дюралевых мерных стержней 1, 4 диаметром 30 мм.

\section{2. Результаты динамических испытаний на сжатие}

Начальная скорость ударника варьировалась в пределах 12-25 м/с. Деформирование образца проходило в несколько циклов, связанных с распространением волн сжатия и растяжения в стержнях. Скорость деформации в экспериментах изменялась в пределах 1500-3500 c $\mathrm{c}^{-1}$. Нагружение проводилось стальным ударником длиной 300 мм. На рис. 5 показаны диаграммы деформирования образцов при сжатии по нормали к слоям сеток, включающие участки активного нагружения и разгрузки 1 - сетка $2 \times 2 \times 0,5$ мм образец из 10 слоев; 2 - сетка $2 \times 2 \times 0,5$ мм образец из 20 слоев; 3 - сетка $3,2 \times 3,2 \times 0,8$ мм образец из 10 слоев; 4 - сетка $3,2 \times 3,2 \times 0,8$ мм образец из 20 слоев. Здесь и далее в качестве меры деформации принимается техническая деформация. Видно, что деформирование при активном нагружении носит ярко выраженный нелинейный характер, в то время как разгрузочные ветви близки к прямым линиям. Погрешность в проведенных экспериментах не превышала 7-10\%. Разгрузочный модуль $E_{r}$ в проведенных испытаниях изменялся от 19 до 26 ГПа. Кривые деформирования слабо зависят от количества слоев, формы образца и типа рассматриваемых сеток. Полученные при активном нагружении динамические кривые деформирования располагаются значительно выше статической кривой (на рис. 5 результаты статических испытаний [14] обозначены пунктиром), что свидетельствует о ярко выраженном отличии деформационных характеристик пакетов плетеных сеток в динамическом и статическом режимах нагружения.

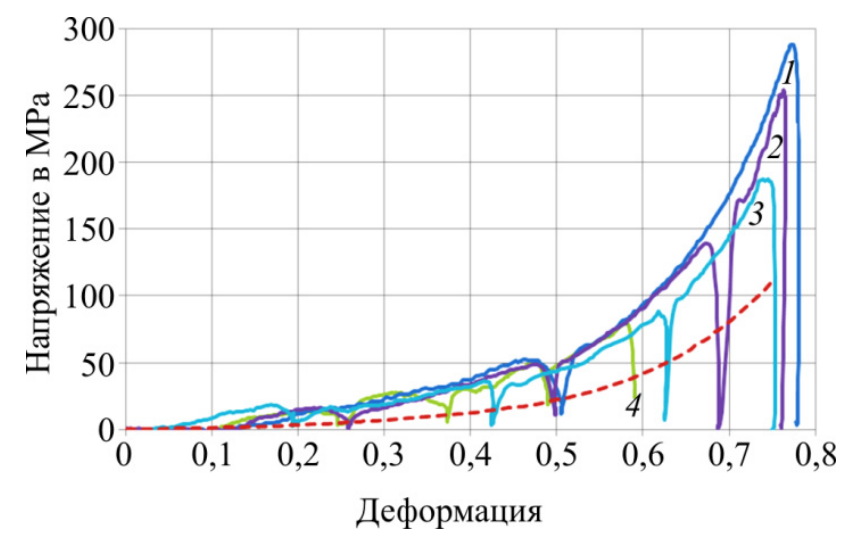

Рис. 5. Диаграммы деформирования на сжатие по нормали к слоям сетки

Fig. 5. Charts of compression deformation normal to grid layers 


\section{3. Результаты динамических испытаний на растяжение}

В экспериментах на растяжение пакетов в плоскости слоев сетки вдоль направления проволок (ось ортотропии) испытывался образец, состоящий из 10 слоев сетки $2 \times 2 \times 0,5$ мм, размеры образца $110 \times 20$ мм. Одной из целей исследования являлось определение зависимости деформационных характеристик на растяжение от предварительного квазистатического сжатия по нормали к слоям сетки. Толщина образца варьировалась в зависимости от предварительного обжатия. Длина рабочей части образца равна 14 мм. На рис. 6 приведен начальный вид образца и образца, разрушенного в процессе ударного растяжения. На рис. 7 показаны усредненные по нескольким опытам диаграммы на растяжение при

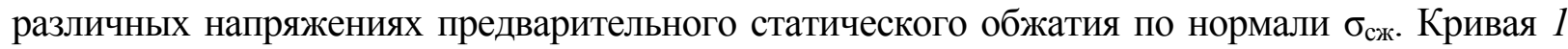
получена при $\sigma_{c ж}=37,5$ МПа, кривая $2-$ при $\sigma_{c ж}=100$ МПа, кривая $3-$ при $\sigma_{\text {сж }}=150 \mathrm{MПа,}$ кривая 4 - при $\sigma_{c ж}=200$ МПа. Результаты проведенного исследования не выявили существенного влияния предварительного обжатия в исследуемом диапазоне, разброс полученных кривых деформирования не превышает разброса кривых деформирования, полученных в одинаковых условиях.
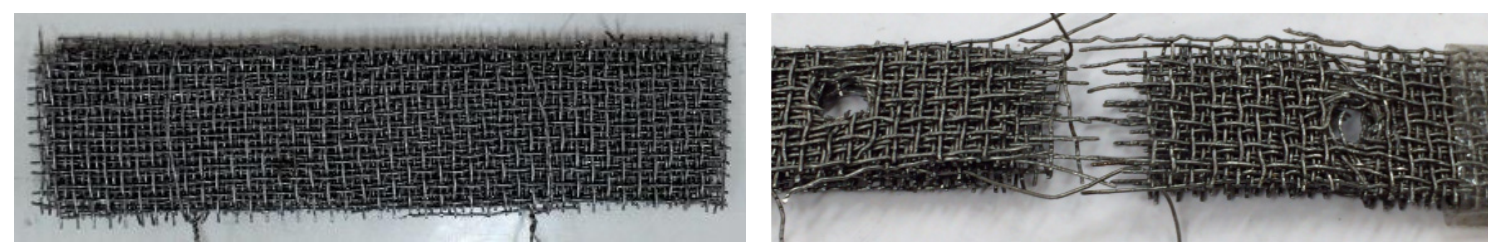

Рис. 6. Образец до и после эксперимента

Fig.6. Sample before and after the experiment

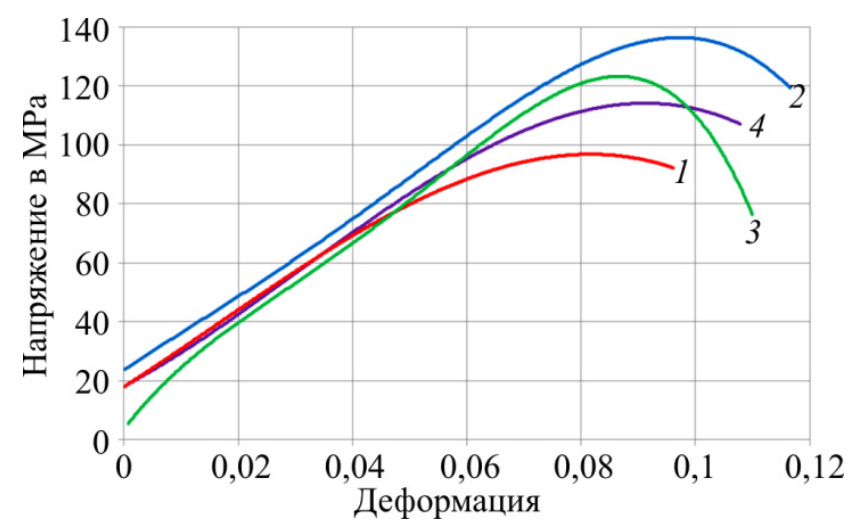

Рис. 7. Диаграммы динамического растяжения вдоль проволок сетки

Fig. 7. Charts of dynamic stressing along the grid wires

\section{4. Результаты статических испытаний на растяжение}

Статические испытания проводились на пакете состоящем из 10 слоев. Размер образца, шаг ячеи и диаметр сечения проволоки такой же, как и при динамических испытаниях на растяжение. Экспериментальные исследования были проведены при длине рабочей части образца 14 мм, что соответствовало условиям проведения динамических испытаний. Образцы, предварительно статически обжатые до напряжений: $1-\sigma_{\text {сж }}=0$ МПа; 
$2-\sigma_{\text {сж }}=5 \mathrm{MПа;} 3-\sigma_{\text {сж }}=25$ МПа; $4-\sigma_{\text {сж }}=50$ МПа; $5-\sigma_{\text {сж }}=150$ МПа, устанавливались в захваты сервогидравлической машины Zwick Z030 [19] и растягивались. В статических экспериментах на растяжение наблюдалась хорошая повторяемость. Разброс не превышал $5 \%$. На рис. 8 показаны характерные кривые при разных уровнях статического обжатия для иллюстрации его сильного влияния. Для одного из образцов при $\sigma_{\text {сж }}=45$ МПа программа испытаний включала поэтапные нагружения и разгрузки, показанные на рис. 9. Видна слабая изменяемость модуля упругости, мало зависящего от степени напряжения образца. Пакеты сеток, как и в случае испытаний на сжатие по нормали, проявляют при растяжении вдоль проволок нелинейные свойства и испытывают необратимые деформации. В отличие от результатов динамических испытаний, квазистатическое деформирование пакетов сеток при растяжении обнаруживает существенную зависимость от степени предварительного обжатия и носит более выраженный нелинейный характер.

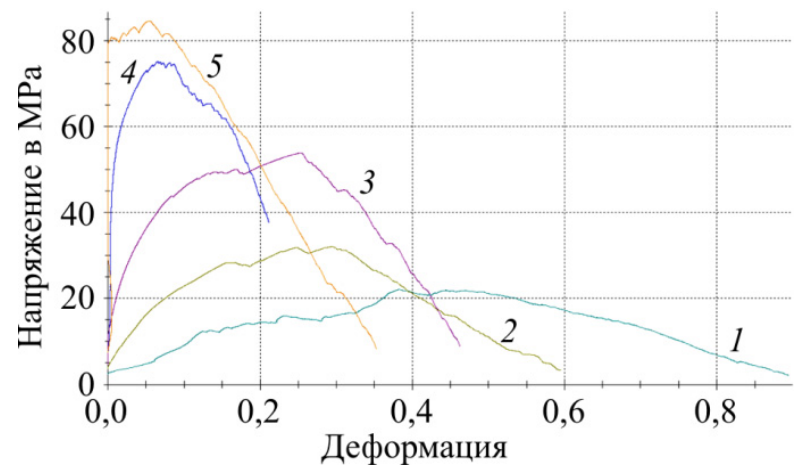

Рис. 8. Диаграммы статического растяжения вдоль проволок сетки

Fig. 8. Charts of static stressing along the grid wires

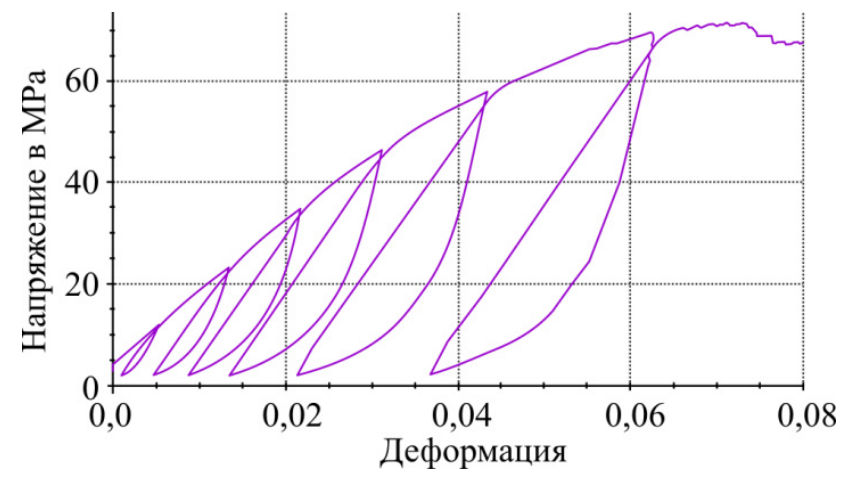

Рис. 9. Диаграмма статического растяжения с разгрузками Fig. 9. Chart of static stretching with unloadings

\section{Заключение}

По результатам динамических испытаний образцов многослойных пакетов плетеных металлических сеток на сжатие по нормали к слоям выявлено существенное отличие динамических диаграмм деформирования от статических. Кривые при всех режимах нагружения носят нелинейный и необратимый характер, то есть при сжатии проявляются пластические свойства.

Диаграммы деформирования на растяжение вдоль оси ортотропии в плоскости слоев также носят нелинейный и необратимый характер вплоть до разрушения образцов, а так- 
же зависят от режима нагружения. При квазистатическом растяжении пакетов сеток вдоль проволок существенное влияние на поведение кривых деформирования оказывает предварительное обжатие пакетов по нормали к слоям сеток.

Работа выполнена при финансовой поддержке РНФ грант № 15-19-10032 в части результатов динамических испытаний на растяжение, грант №16-19-10113 в части статических и динамических испытаний на сжатие

\section{Библиографический список}

1. Stolz A., Ruiz-Ripoll M.L. Experimental and computational characterization of dynamic loading and structural resistance of tunnels in blast scenarios // Fire technology. - 2015. - P. 24. DOI: $10.1007 / \mathrm{s} 10694-015-0496-8$

2. An Inverse Estimation of High Strain Rate Properties of Composite Material Constituents / S. Chacko, A. Jones, R. Brooks, M.J. Lidgett // 20th International Conference on Composite Materials Copenhagen, 19-24th July 2015, Copenhagen, 2015.

3. Splichal J., Pistek A., Hlinka J. Dynamic tests of composite panels of an aircraft wing // Progress in Aerospace Sciences. 06/2015. DOI:10.1016/j.paerosci.2015.05.005

4. Cadoni E, Forni D. Strain rate effects on reinforcing steels in tension // EPJ Web of Conferences 94 01004, 2015. DOI: http://dx.doi.org/10.1051/epjconf/20159401004

5. Zhu H, Pierron F. Exploration of Saint-Venant's Principle in Inertial High Strain Rate Testing of Materials // Experimental Mechanics. - 07/2015. DOI: 10.1007/s11340-015-0078-1.

6. Hu D., Meng K., Jiang H. Experimental Investigation of Dynamic Properties of AerMet 100 Steel // Procedia Engineering - 12/2015; 99:1459-1464. DOI:10.1016/j.proeng.2014.12.685

7. Гельфанд Б.Е., Сильников М.В. Фугасные эффекты взрывов. - СПб.: Полигон, 2002. - 266 с.

8. Альтшулер Л.В., Кругликов Б.С. Затухание сильных ударных волн в двухфазных и гетерогенных средах // ПМТФ. - 1984. - № 5. - С. 24-29.

9. Кругликов Б.С., Кутушев А.Г. Ослабление ударных волн экранирующими решетками // ФГВ. - 1998. - Т. 24, № 1. - С. 115-118.

10. Численная модель деформирования противоосколочной сетки при взрывном нагружении / А.И. Абакумов [и др.] // Тр. ВНИИЭФ. Математическое моделирование физических процессов. 2006. - № 10. - С. 16-30.

11. Моделирование взаимодействия ударных волн с деформируемыми газопроницаемыми преградами / М.Х. Абузяров, Е.Г. Глазова, А.В. Кочетков, С.В. Крылов, В.И. Романов, М.А. Сырунин // Проблемы прочности и пластичности. - 2010. - Вып. 72. - С. 120-129.

12. Мельцас В.Ю., Портнягина Г.Ф., Соловьев В.П. Численное моделирование прохождения ударных волн через экранирующие решетки // ВАНТ. - 1993. Вып. 3. - С. 26-31.

13. Глазова Е.Г., Кочетков А.В. Численное моделирование взаимодействия деформируемых газопроницаемых пакетов сеток с ударными волнами // ПМТФ. - 2012. - № 3. - С. 11-19.

14. Исследование деформационных свойств пакетов плетеных металлических сеток при квазистатическом сжатии и растяжении / А.Н. Горохов, Д.А. Казаков, А.В. Кочетков, И.А. Модин, В.И. Романов // Проблемы прочности и пластичности. - 2014. - Вып. 76 (3). - С. 251-255.

15. Bragov A.M., Lomunov A.K. Methodological aspects of studying dynamic material properties using the Kolsky method // Int.J. of Impact Engineering. - 1995. - Vol. 16 (2). - P. 321-330.

16. Investigations on specimen design and mounting for Split Hopkinson Tension Bar (SHTB) experiments / N. Ledford, H. Paul, G. Ganzenmüller, M. May, M. Höfemann, M. Otto, N. Petrinic // DYMAT. - 09/2015. 
17. Design and Computational Validation of a Split Hopkinson Pressure Bar for Dynamic Characterization of Materials Under High Strain Rate Tension Loading / A. Sasikumar, N. John, S. Pushpagiri, L.L. Koithara // International Journal of Engineering Research \& Technology. - 2015. Vol. 4 - Iss. 06, June. DOI: http://dx.doi.org/10.17577/IJERTV4IS060894

18. Nicholas O. Tensile testing of materials at high rates of strain // Exp.Mech. - 1981. - Vol. 21. No. 5. - P. 177-195.

19. Методика определения параметров уравнений механики поврежденной среды при усталости и ползучести / И.А. Волков, Д.А. Казаков, Ю.Г. Коротких, А.И. Волков // Прикладная механика и технологии машиностроения. - 2012 - № 2 (21) - С. 7-24.

\section{References}

1. Stolz A., Ruiz-Ripoll M.L. Experimental and computational characterization of dynamic loading and structural resistance of tunnels in blast scenarios. Fire technology, 2015, pp. 24-30. DOI: 10.1007/s10694-015-0496-8

2. Chacko S., Jones A., Brooks R., Lidgett M.J. An Inverse Estimation of High Strain Rate Properties of Composite Material Constituents. 20th International Conference on Composite Materials Copenhagen, 19-24th July 2015, Copenhagen.

3. Splichal J., Pistek A., Hlinka J. Dynamic tests of composite panels of an aircraft wing. Progress in Aerospace Sciences - 06/2015. DOI: 10.1016/j.paerosci.2015.05.005

4. Cadoni E, Forni D. Strain rate effects on reinforcing steels in tension. EPJ Web of Conferences 9401004 - 2015. DOI: http://dx.doi.org/10.1051/epjconf/20159401004

5. Zhu H, Pierron F. Exploration of Saint-Venant's Principle in Inertial High Strain Rate Testing of Materials. Experimental Mechanics, 07/2015. DOI: 10.1007/s11340-015-0078-1

6. Hu D., Meng K., Jiang H. Experimental Investigation of Dynamic Properties of AerMet 100 Steel, Procedia Engineering, 12/2015; 99: 1459-1464. DOI: 10.1016/j.proeng.2014.12.685

7. Gelfand B.E., Silnikov M.V. Fugasnye effekty vzryvov [High-explosive explosion effects]. Sankt-Peterburg. OOO «Izdatel'stvo «Poligon», 2002, 266 p.

8. Altshuler L.V., Kruglikov B.S. Zatukhanie sil'nykh udarnykh voln v dvukhfaznykh i geterogennykh sredakh [Attenuation of strong shock waves in a two-phase and heterogeneous environments]. PMTF, 1984, no. 5, pp. 24-29.

9. Kruglikov B.S., Kutushev A.G. Oslablenie udarnykh voln ekraniruiushchimi reshetkami [Attenuation of shock waves Louvre]. FGV, 1998, vol. 24, no. 1, pp. 115-118.

10. Abakumov A.I. [Et al.] Chislennaia model' deformirovaniia protivooskolochnoi setki pri vzryvnom nagruzhenii [Numerical model of anti-shatter deformation grid under explosive loading]. Tr. VNIIEF. Matematicheskoe modelirovanie fizicheskikh protsessov, 2006, no. 10, pp. 16-30.

11. Abuzyarov M.H., Glazov E.G., Kochetkov A.V., Krylov S.V., Romanov V.I., Syrunin M.A. Modelirovanie vzaimodeistviia udarnykh voln $\mathrm{s}$ deformiruemymi gazopronitsaemymi pregradami [Modeling of shock waves interaction with deformable gas-permeable barriers]. Problemy prochnosti $i$ plastichnosti, 2010, is. 72, pp. 120-129.

12. Meltsas V.Y., Portnyagina G.F., Solovyov V.P. Chislennoe modelirovanie prokhozhdeniia udarnykh voln cherez ekraniruiushchie reshetki [Numerical simulation of the passage of shock waves through the louvres]. VANT, 1993, is. 3, pp. 26-31.

13. Glazov E.G., Kochetkov A.V. Chislennoe modelirovanie vzaimodeistviia deformiruemykh gazopronitsaemykh paketov setok s udarnymi volnami [Numerical simulation of the deformable mesh breathable packages with shock waves]. PMTF, 2012, no. 3, pp. 11-19.

14. Gorokhov A.N., Kazakov D.A., Kochetkov A.V., Modin I.A., Romanov V.I. Issledovanie deformatsionnykh svoistv paketov pletenykh metallicheskikh setok pri kvazistaticheskom szhatii i 
rastiazhenii [The study of deformation properties of a package of woven metal mesh under quasi-static compression and tension]. Problemy prochnosti i plastichnosti, 2014, is. 76 (3), pp. 251-255.

15. Bragov A.M., Lomunov A.K. Methodological aspects of studying dynamic material properties using the Kolsky metho. Int.J. of Impact Engineering, 1995, no. 16 (2), pp. 321-330.

16. Ledford N., Paul H., Ganzenmüller G, May M., Höfemann M., Otto M., Petrinic N. Investigations on specimen design and mounting for Split Hopkinson Tension Bar (SHTB) experiments. DYMAT, 09/2015.

17. Sasikumar A., John N., Pushpagiri S., Koithara L.L. Design and Computational Validation of a Split Hopkinson Pressure Bar for Dynamic Characterization of Materials Under High Strain Rate Tension Loading. International Journal of Engineering Research \& Technology, vol. 4, is. 06, June - 2015 e-ISSN: 2278-0181. DOI: http://dx.doi.org/10.17577/IJERTV4IS060894

18. Nicholas O. Tensile testing of materials at high rates of strain. Exp.Mech, 1981, vol. 21, no. 5, pp. 177-195.

19. Volkov I.A., Kazakov D.A., Korotkikh Y.G., Volkov A.I. Metodika opredeleniia parametrov uravnenii mekhaniki povrezhdennoi sredy pri ustalosti i polzuchesti [Method for determining the parameters of the equations of mechanics damaged environment for fatigue and creep]. Prikladnaia mekhanika i tekhnologii mashinostroeniia, 2012, no. 2 (21), pp. 7-24. 